\title{
PERCEPCIÓN DE MÉDICOS QUE REALIZAN EL SERVICIO RURAL Y URBANO-MARGINAL DE SALUD EN PERÚ SOBRE EL PRIMER NIVEL DE ATENCIÓN
}

\author{
Guido Bendezu-Quispe (1) ${ }^{1, a}$, Luis Felipe Mari-Huarache (ib ${ }^{2, b}$, Álvaro Taype-Rondan (1) 1,c, \\ Christian R. Mejia ${ }^{3, \mathrm{~d}}$, Fiorella Inga-Berrospi (i) $4, \mathrm{e}$ \\ ${ }^{1}$ Universidad San Ignacio de Loyola, Vicerrectorado de Investigación, Unidad de Investigación para la Generación y Síntesis \\ de Evidencias en Salud, Lima, Perú. \\ 2 Universidad Ricardo Palma, Lima, Perú. \\ 3 Universidad Continental, Lima, Perú. \\ ${ }^{4}$ Universidad Privada Norbert Wiener, Lima, Perú. \\ ${ }^{a}$ Médico cirujano, magíster en Informática Biomédica; ${ }^{\mathrm{b}}$ estudiante de medicina humana; ${ }^{\mathrm{c}}$ médico cirujano, magíster en \\ Epidemiología; ${ }^{\circledR}$ médico cirujano, doctor en Medicina Clínica y Traslacional; ${ }^{\mathrm{e}}$ médico cirujano, especialista en Gestión en \\ Salud.
}

\section{RESUMEN}

Objetivo: Determinar la percepción de los médicos que realizan el Servicio Rural y Urbano Marginal de Salud (SERUMS) en Perú sobre el trabajo en el primer nivel de atención (PNA) y sus factores asociados. Materiales y métodos: Se realizó un análisis secundario de datos de una muestra de médicos que realizaron el SERUMS el 2016. Para evaluar la variable de interés se utilizó la escala de «Percepción sobre el trabajo en el PNA», cuyos puntajes más altos indican una peor percepción del trabajo en el PNA. Se aplicó una encuesta basal (antes del SERUMS) y una encuesta de seguimiento (8-12 meses de iniciado el SERUMS) y se evaluaron las diferencias en los puntajes. Resultados: De los 780 encuestados, 215 (27,6\%) completaron la encuesta basal y de seguimiento. La media del puntaje incrementó considerablemente (de 3,4 a 6,7; $\mathrm{p}<0,001)$, lo que indica una peor percepción del trabajo en el PNA tras iniciar el SERUMS. De los tres dominios de la encuesta, el de las percepciones sobre el médico que trabaja en el PNA y el de las percepciones sobre el trabajo asistencial en el PNA incrementaron los puntajes de percepción sobre el trabajo en el PNA. No se encontraron variables sociodemográficas asociadas al cambio en los puntajes. Conclusiones: La percepción de los médicos sobre el trabajo en el PNA se deteriora luego de iniciar el SERUMS. Se deben promover estrategias que incentiven el interés de los médicos en este nivel de atención.

Palabras clave: Atención Primaria de Salud; Médicos de Atención Primaria; Salud Rural; Servicios de Salud Rural; Comunidades Rurales; Servicios de Salud Suburbana; Personal de Salud; Perú (Fuente: DeCS BIREME).

\section{EFFECT OF RURAL AND MARGINAL URBAN HEALTH SERVICE ON THE PHYSICIANS' PERCEPTION OF PRIMARY HEALTH CARE IN PERU}

Citar como: Bendezu-Quispe G, MariHuarache LF, Taype-Rondan A, Mejia CR, Inga-Berrospi F. Percepción de los médicos que realizan el Servicio Rural y Urbano-Marginal de Salud en Perú sobre el primer nivel de atención. Rev Peru Med Exp Salud Publica. 2020;37(4):63644. doi: https://doi.org/10.17843/ rpmesp.2020.374.5294

Correspondencia: Guido BendezuQuispe; Universidad San Ignacio de Loyola, Av. La Fontana 550, La Molina, Lima, Perú; gbendezu@usil.edu.pe

Recibido: $25 / 02 / 2020$ Aprobado: 08/07/2020 En línea: $12 / 10 / 2020$

\begin{abstract}
Objective: To determine the effect of the Rural and Marginal Urban Health Service (SERUMS) on the physicians' perception of work in the primary health care (PHC) setting and its associated factors. Materials and methods: A secondary data analysis of a sample of physicians who performed the SERUMS in 2016 was carried out. To evaluate the variable of interest, the scale "Perception of work in the PHC setting" was used, higher scores indicated a negative perception of work in the PHC setting. A baseline survey (before the SERUMS) and a follow-up survey (8-12 months after starting the SERUMS) were applied and differences in both scores were evaluated. Results: Of the 780 respondents, 215 (27.6\%) completed the baseline and follow-up survey. The average score increased considerably (from 3.4 to $6.7 ; \mathrm{p}<0.001$ ), which shows a negative perception of work in the PHC setting after participating in the SERUMS. Of the three parts of the survey, the one regarding perceptions by the physicians working in the PHC and the one about perceptions of medical work in the PHC setting increased the perception scores. No sociodemographic variables were found to be associated with the change in scores. Conclusions: Physicians' perception about work in the PHC setting deteriorated after participating in the SERUMS. Therefore, strategies to encourage physicians' interest in working at this level of healthcare should be promoted.
\end{abstract}

Keywords: Primary Health Care; Physicians; Primary Care; Rural Health; Rural Health Services; Rural settlements; Suburban Health Services; Health Personnel; Peru (Source: MeSH NLM). 


\section{INTRODUCCIÓN}

Para satisfacer las necesidades de salud de la población se requiere de profesionales calificados, disponibles y distribuidos de forma equitativa. En los últimos años, se ha incrementado el número de profesionales de la salud en todo el mundo; sin embargo, en el $40 \%$ de los países todavía existen menos de 10 médicos por cada 10000 personas, situación que acontece en el 90\% de los países de bajos ingresos ${ }^{(1)}$. En el 2017, el Perú tenía 12,8 médicos por cada 10000 habitantes, y se estimaba que solo 7641 médicos (la tercera parte del total) laboraban en el primer nivel de atención (PNA) ${ }^{(2)}$, un porcentaje bajo, considerando que el PNA debería resolver el $80 \%$ de los problemas de salud de la población ${ }^{(3)}$.

En 1972, se crea en el Perú el Servicio Civil de Graduandos (SECIGRA), actualmente denominado Servicio Rural y Urbano Marginal de Salud (SERUMS), un programa comunitario realizado por los profesionales de la salud durante un año en el PNA. Está orientado a mejorar la distribución de profesionales de la salud en todo el país, a fin de brindar el acceso a la atención integral de la salud a la población vulnerable de las zonas rurales y urbano marginales del Perú ${ }^{(4,5)}$. Si bien en los últimos años se ha incrementado el número de profesionales de la salud en el PNA ${ }^{(5)}$, incluyendo a los serumistas ${ }^{(6)}$, se ha reportado un bajo interés de los médicos por laborar en este nivel de atención ${ }^{(7-10)}$.

El SERUMS representa la primera experiencia de trabajo en el PNA para muchos profesionales de la salud, esto podría influir significativamente en la percepción y en la intención de laborar en el PNA en el futuro. Entender esta situación sería de utilidad para mejorar las políticas de atracción hacia el trabajo en este nivel de atención. Por ello, el objetivo del presente estudio fue determinar la percepción de los médicos que realizan el SERUMS en Perú sobre el trabajo en el primer nivel de atención y sus factores asociados.

\section{MATERIALES Y MÉTODOS}

\section{Diseño y población de estudio}

Se hizo un análisis secundario con los datos procedentes de las encuestas del Colegio Médico del Perú (CMP) a un grupo de médicos antes de iniciar el SERUMS y en el octavo mes de servicio.

\section{Fuentes de información}

El Comité del Médico Joven del CMP realizó dos encuestas en el 2016 con el objetivo de obtener información sobre las perspectivas profesionales hacia el trabajo en el PNA.

\section{Encuesta basal}

En abril del 2016, durante el «Curso de Inducción SERUMS Lima, 2016» organizado por el Comité del Médico Joven del Consejo Nacional del CMP y el Consejo Regional III-Lima,

\section{MENSAJES CLAVE}

Motivación para realizar el estudio: El Servicio Rural y Urbano Marginal de Salud (SERUMS) suele ser la primera experiencia de trabajo en el primer nivel de atención (PNA) de los médicos recién egresados en el Perú.

Principales hallazgos: La percepción de los médicos sobre el trabajo en el PNA se deteriora luego de 8 a 10 meses en el SERUMS. El nivel básico de quechua y aimara, y tener una familia dependiente se asocian a una mejor percepción de trabajo en el PNA.

Implicancias: Las estrategias para fomentar el interés del médico serumista en el PNA deben incluir nuevos incentivos para esta actividad, de tipo mixto (económicos y no económicos).

se encuestó a los médicos que estaban próximos a realizar el SERUMS. Este evento tuvo como finalidad brindar las herramientas y el conocimiento para el desarrollo de las funciones durante el SERUMS, con énfasis en el trabajo en el PNA.

\section{Encuesta de seguimiento}

Del octavo al duodécimo mes, luego de iniciado el SERUMS, se aplicó una segunda encuesta a los médicos que habían completado la encuesta basal. Esta se creó en Google Forms ${ }^{\otimes}$ y se envió por correo electrónico. Adicionalmente, se llamó por teléfono a los participantes para recordarles el llenado del cuestionario. Los correos electrónicos y los números telefónicos se obtuvieron de la base de datos proporcionada por el CMP.

\section{Variable dependiente}

Se consideró como variable dependiente a la percepción sobre el trabajo en el PNA, la cual se midió con una escala validada ${ }^{(11)}$. Este instrumento tiene 11 ítems, cuyas respuestas se encuentran en escala Likert, de uno a cinco puntos. El estudio que ha validado esta escala reporta una consistencia interna global alta (alfa $=0,78$; puntaje para cada ítem mayor a 0,7), y una correlación entre los dominios superior a 0,3 , en todos los casos ${ }^{(11)}$.

La escala tiene una puntuación global que va de 11 a 55 puntos (siendo 11 el valor de mayor percepción positiva sobre el trabajo en el PNA y el de 55 el de mayor percepción negativa sobre el trabajo en el PNA). Además, presenta tres dominios: «Percepciones sobre el médico que trabaja en el PNA» (rango de 5 a 25); «Percepciones sobre el trabajo asistencial en el PNA» (rango de 4 a 20); y «Percepciones sobre las consecuencias económicas de trabajar en el PNA» (rango de 2 a 10). Los puntajes más altos indican una peor percepción sobre el trabajo en el PNA ${ }^{(11)}$. 


\section{Variables independientes}

En la encuesta basal se incluyeron al sexo, edad, universidad de procedencia, lugar de nacimiento, haber estudiado una carrera previa, tener un nivel básico de quechua o aimara, tener una familia dependiente (principal generador de ingreso económico familiar), tener familiares médicos, y haber participado en actividades en el PNA durante el pregrado médico. En la encuesta de seguimiento, se consultó sobre el tipo de establecimiento donde realizaron el SERUMS, la categoría del establecimiento de salud y sobre la posibilidad de jefatura del establecimiento de salud.

\section{Plan de análisis}

Se procesó y analizó la base de datos de la encuesta basal, con el programa estadístico Stata v14.0 (Stata Corporation, College Station, Texas, EE. UU.). Las características generales de los médicos se resumieron en frecuencias absolutas y relativas para las variables categóricas, así como medidas de tendencia central y dispersión para las variables numéricas. Para todos los análisis se consideró un valor de $\mathrm{p}<0,05$ como estadísticamente significativo. Para comparar los puntajes entre la encuesta basal y la de seguimiento, se usó la prueba t para muestras pareadas.

Para evaluar los factores asociados a la percepción sobre el trabajo en el PNA y los factores asociados al cambio de puntaje entre la encuesta basal y la de seguimiento, se usaron regresiones lineales crudas y ajustadas, calculando los coeficientes $\beta$ y sus intervalos de confianza al 95\% (IC 95\%). En el modelo ajustado, se incluyeron las variables que presentaron un valor de $\mathrm{p}<0,20$ en el modelo crudo. Para realizar estas regresiones lineales, se evaluó previamente el cumplimiento de los criterios de normalidad de residuos (evaluación visual de los histogramas de residuos estudentizados) y varianzas iguales (evaluación visual del gráfico de dispersión entre los residuos y los valores predichos del desenlace). Al ser la variable independiente categórica, se determinó que las regresiones cumplían con el supuesto de linealidad. En todos los casos se cumplió con los supuestos de la regresión lineal múltiple. Asimismo, en el modelo ajustado se usaron factores de inflación de la varianza no centrada; no se encontró colinealidad entre las variables independientes.

\section{Consideraciones éticas}

La participación de los médicos fue voluntaria. Se contó con la aprobación del Comité de Ética Institucional del Hospital Nacional Docente Madre Niño San Bartolomé (Exp. N.: 09400-19) para la realización de este estudio.

\section{RESULTADOS}

Fueron 780 médicos quienes respondieron la encuesta basal. De ellos, 723 completaron la escala de percepción del trabajo en el PNA, de los cuales 215 (29,7\%) completaron la encuesta de seguimiento (Figura 1 ).

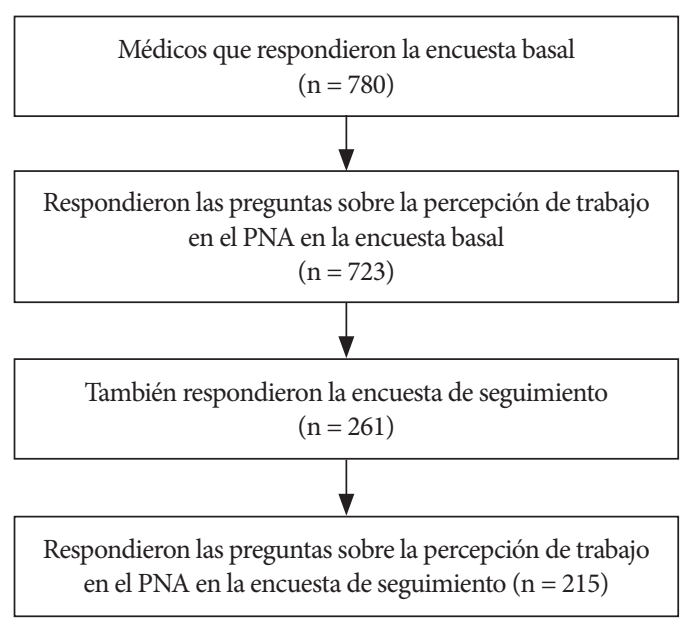

Figura 1. Flujograma de los médicos incluidos en el estudio.

En los 723 médicos que completaron la escala de percepción del trabajo en el PNA en la encuesta basal, la mediana de la edad fue 25 años, 389 (53,8\%) fueron mujeres, $627(86,8 \%)$ estudiaron en una escuela de medicina de Lima, $84(12,8 \%)$ tuvieron familia que dependía de ellos para su subsistencia (Tabla 1 ).

En la Tabla 2 se presenta la comparación de los puntajes de la escala de percepción sobre el trabajo en el PNA, para la encuesta basal $(n=723)$ y la de seguimiento $(n=215)$. Los 11 ítems evaluados presentaron diferencias estadísticamente significativas $(p<0,05)$. En general, se incrementaron los puntajes, es decir, la percepción del trabajo en el PNA empeoró luego de ocho meses de iniciado el SERUMS, excepto el puntaje del ítem 4 (¿considera que un médico que labora en el PNA tiene un menor ingreso económico que un médico que labora en un hospital?), cuyo puntaje descendió. Los mayores puntajes se presentaron en los ítems 1, 2, 7 y 9. Asimismo, se encontraron diferencias estadísticamente significativas $(\mathrm{p}<0,005)$ en dos de las tres dimensiones, excepto en la dimensión «Percepciones sobre las consecuencias económicas de trabajar en el PNA».

Los siguientes ítems presentaron los más altos puntajes: 11 (¿considera que la formación académica que ha recibido en la universidad está orientada más a un trabajo hospitalario que del PNA?), 10 (¿considera que el trabajo en el PNA es muy limitado, en relación con la actividad hospitalaria?), 6 (¿considera que si trabajase en el PNA en su país, no podría satisfacer sus necesidades económicas?) y 4 (¿usted considera que un médico que labora en el PNA tiene un menor ingreso económico que un médico que labora en un hospital?).

En el modelo ajustado de la encuesta basal, se encontró que los médicos con un nivel básico de quechua o aimara tuvieron en promedio 2,67 (IC 95\%: -4,29 a -1,06) puntos menos que aquellos que no tenían conocimiento de quechua o aimara; y aquellos que tenían una familia dependiente tuvieron en promedio 1,75 (IC 95\%: -3,48 a -0,01) puntos 
Tabla 1. Características de los médicos participantes del estudio.

\begin{tabular}{|c|c|}
\hline Variables & $\begin{array}{c}\text { Total } \\
(\mathrm{n}=723)\end{array}$ \\
\hline Edad (años) ${ }^{a}$ & $25(24-27)$ \\
\hline \multicolumn{2}{|l|}{ Sexo } \\
\hline Masculino & $334(46,2)$ \\
\hline Femenino & $389(53,8)$ \\
\hline \multicolumn{2}{|c|}{ Ubicación de la universidad de procedencia } \\
\hline Ciudad de Lima & $627(86,8)$ \\
\hline Otras ciudades del Perú & $80(11,1)$ \\
\hline Extranjero & $15(2,1)$ \\
\hline \multicolumn{2}{|l|}{ Lugar de nacimiento } \\
\hline Ciudad de Lima & $429(59,3)$ \\
\hline Otras ciudades del Perú & $286(39,6)$ \\
\hline Extranjero & $8(1,1)$ \\
\hline \multicolumn{2}{|c|}{ Estudió otra carrera antes de Medicina } \\
\hline No & $659(91,9)$ \\
\hline Sí & $58(8,1)$ \\
\hline \multicolumn{2}{|c|}{ Nivel básico de quechua o aimara } \\
\hline No & $500(85,6)$ \\
\hline Sí & $84(14,4)$ \\
\hline \multicolumn{2}{|l|}{ Familia dependiente } \\
\hline No & $620(87,6)$ \\
\hline Sí & $88(12,4)$ \\
\hline \multicolumn{2}{|l|}{ Familiares médicos } \\
\hline No & $372(51,7)$ \\
\hline Sí & $348(48,3)$ \\
\hline \multicolumn{2}{|c|}{$\begin{array}{l}\text { Participación en actividades de PNA durante } \\
\text { el pregrado }\end{array}$} \\
\hline No & $40(5,5)$ \\
\hline Sí & $683(94,5)$ \\
\hline \multicolumn{2}{|l|}{ Variables del SERUMS ${ }^{\text {b }}$} \\
\hline \multicolumn{2}{|c|}{ Tipo de establecimiento de salud } \\
\hline Ministerio de Salud & $232(89,2)$ \\
\hline Otros & $28(10,8)$ \\
\hline \multicolumn{2}{|c|}{ Categoría del establecimiento de salud } \\
\hline $\mathrm{I}-1$ & $44(16,9)$ \\
\hline $\mathrm{I}-2$ & $148(56,7)$ \\
\hline I-3 o I-4 & $69(26,4)$ \\
\hline \multicolumn{2}{|c|}{ Jefatura del establecimiento de salud } \\
\hline No & $168(64,4)$ \\
\hline Sí & $93(35,6)$ \\
\hline
\end{tabular}

PNA: Primer nivel de atención.

${ }^{a}$ Mediana (rango intercuartílico).

b Solo en aquellos médicos que respondieron la encuesta de seguimiento.

menos que aquellos que no tenían una familia dependiente, ajustado por edad, universidad de procedencia, lugar de nacimiento, otras carreras universitarias y participación en actividades de PNA durante el pregrado. El modelo ajustado tuvo un valor de $\mathrm{R}^{2}$ de 0,034 , es decir, la adición de todas las variables de este modelo explica el 3,4\% de la variabilidad de la percepción por trabajar en el PNA (Tabla 3).

En cuanto a los factores asociados al cambio de puntaje entre la percepción en la encuesta basal y la de seguimiento, en el modelo de regresión lineal simple, ninguna varia- ble presentó una asociación estadísticamente significativa al cambio, por lo que no se realizó el modelo de regresión lineal múltiple (Tabla 4).

\section{DISCUSIÓN}

Los resultados del presente estudio indican que la percepción de los serumistas sobre el trabajo en el PNA empeora luego de un periodo de 8-12 meses. Este hallazgo se presentó en la puntuación global y en los dominios «Percepciones sobre el médico que trabaja en el PNA» $\mathrm{y}$ «Percepciones sobre el trabajo asistencial en el PNA». A excepción del ítem ¿Usted considera que un médico que labora en el PNA tiene un menor ingreso económico que un médico que labora en un hospital?, cuya percepción mejoró. Asimismo, los factores sociodemográficos no se asociaron al cambio de puntaje en la percepción sobre el trabajo en el PNA.

Los resultados indican que la percepción sobre el trabajo en el PNA se deteriora conforme pasan los meses, lo que señalaría la necesidad de mejoras en la experiencia laboral de los participantes de este programa. Los médicos peruanos tienen poco interés en trabajar en el PNA ${ }^{(10,12)}$, lo cual se debería a la falta de incentivos económicos y no económicos, $\mathrm{y}$ a su mayor interés por la labor hospitalaria ${ }^{(7-10)}$.

Los médicos que manifestaron que su formación académica estuvo orientada al ámbito hospitalario, tuvieron una peor percepción hacia el trabajo en el PNA. Según los estudios previos realizados, menos del $50 \%$ de médicos peruanos recientemente graduados consideran que han recibido una capacitación adecuada para el trabajo en el PNA ${ }^{(13)}$, usualmente mediante la participación en asociaciones estudiantiles que realizan actividades sociales ${ }^{(14,15)}$. Esto podría causar que el médico considere la labor del PNA como inferior y menos interesante que la hospitalaria, ya que su formación en las escuelas de medicina estaría orientada a resolver problemas complejos de salud que requieren de establecimientos con mayor capacidad resolutiva.

Por otro lado, se encontró que tener un nivel básico de quechua o aimara, y tener una familia dependiente estuvieron asociados a una mejor percepción del trabajo en el PNA. Estudios previos han descrito que el haber realizado estudios en el interior del país o en zonas rurales ${ }^{(16-18)}$, conocer el idioma quechua, y que la familia proceda del interior del país, se asocian a la posibilidad de trabajar en zonas remotas o rurales ${ }^{(16)}$. Estos escenarios incrementarían la familiaridad de los médicos serumistas con el quechua o aimara y con la labor médica en zonas rurales, lo que explicaría este hallazgo. Respecto a la asociación entre tener una familia dependiente y el trabajo en el PNA, participar en el SERUMS permite al profesional recién egresado recibir un salario, incluso mayor al que obtendría trabajando en la zona urbana, donde existe mayor competencia por un puesto de trabajo en función de la experiencia profesional, pudiendo este ingreso económico 
Tabla 2. Puntaje obtenido por los médicos que respondieron la encuesta basal $(n=723)$ y la de seguimiento $(n=215)$ de la encuesta de percepción sobre el trabajo en el primer nivel de atención.

\begin{tabular}{|c|c|c|c|c|c|}
\hline \multirow{2}{*}{ Preguntas } & \multirow{2}{*}{$\begin{array}{l}\text { Encuesta basal } \\
\qquad(\mathbf{n}=723)\end{array}$} & \multicolumn{4}{|c|}{ Encuesta de percepción de trabajo en el PNA $(n=215)$} \\
\hline & & Basal & Seguimiento & Diferencia & Valor de $\mathrm{p}^{\mathrm{a}}$ \\
\hline $\begin{array}{l}\text { 1. ¿Usted cree que un médico que trabaja en el } \\
\text { PNA tiene menos prestigio ante la sociedad que un } \\
\text { médico que trabaja en un hospital? }\end{array}$ & $2,3(1,1)$ & $2,3(1,1)$ & $2,8(1,2)$ & $0,5(1,4)$ & $<0,001$ \\
\hline $\begin{array}{l}\text { 2. ¿Usted considera que el médico que trabaja en el } \\
\text { PNA es porque no tuvo otra opción laboral? }\end{array}$ & $2,3(0,9)$ & $2,3(1,0)$ & $2,8(1,1)$ & $0,5(1,2)$ & $<0,001$ \\
\hline $\begin{array}{l}\text { 3. ¿Usted considera que el médico que trabaja en } \\
\text { el PNA tiene menor preparación académica que el } \\
\text { que trabaja en un hospital? }\end{array}$ & $2,2(1,0)$ & $2,3(1,0)$ & $2,6(1,0)$ & $0,3(1,2)$ & $<0,001$ \\
\hline $\begin{array}{l}\text { 4. ¿Usted considera que un médico que labora en } \\
\text { el PNA tiene un menor ingreso económico que un } \\
\text { médico que labora en un hospital? }\end{array}$ & $3,4(1,0)$ & $3,5(1,0)$ & $3,2(1,2)$ & $-0,3(1,4)$ & 0,002 \\
\hline $\begin{array}{l}\text { 5. ¿Usted considera que el médico que trabaja en } \\
\text { el PNA tiene un menor estatus dentro del gremio } \\
\text { médico, en comparación con los que trabajan en } \\
\text { un hospital? }\end{array}$ & $2,6(1,1)$ & $2,7(1,1)$ & $3,0(1,1)$ & $0,4(1,3)$ & $<0,001$ \\
\hline $\begin{array}{l}\text { 6. ¿Usted considera que si trabajase en el PNA } \\
\text { en su país, no podría satisfacer sus necesidades } \\
\text { económicas? }\end{array}$ & $3,4(1,1)$ & $3,5(1,0)$ & $3,7(1,1)$ & $0,2(1,2)$ & 0,028 \\
\hline $\begin{array}{l}\text { 7. ¿Para usted el trabajo en el PNA es un período } \\
\text { de transición entre el terminar la carrera y la } \\
\text { especialización (residencia)? }\end{array}$ & $3,3(1,0)$ & $3,4(1,0)$ & $3,9(1,0)$ & $0,5(1,2)$ & $<0,001$ \\
\hline $\begin{array}{l}\text { 8. ¿Usted considera que en el PNA se ven casos } \\
\text { médicos poco interesantes, en relación con la } \\
\text { actividad hospitalaria? }\end{array}$ & $3,0(1,0)$ & $3,1(1,0)$ & $3,4(1,1)$ & $0,3(1,4)$ & 0,002 \\
\hline $\begin{array}{l}\text { 9. ¿Usted considera que el trabajo en el PNA es } \\
\text { rutinario, en relación con la actividad hospitalaria? }\end{array}$ & $3,0(1,0)$ & $3,1(0,9)$ & $3,6(1,0)$ & $0,5(1,2)$ & $<0,001$ \\
\hline $\begin{array}{l}\text { 10. ¿Usted considera que el trabajo en el PNA } \\
\text { es muy limitado, en relación con la actividad } \\
\text { hospitalaria? }\end{array}$ & $3,4(1,0)$ & $3,5(1,0)$ & $3,8(0,9)$ & $0,4(1,1)$ & $<0,001$ \\
\hline $\begin{array}{l}\text { 11. ¿Usted considera que la formación académica } \\
\text { que ha recibido en la universidad está orientada } \\
\text { más a un trabajo hospitalario que del PNA? }\end{array}$ & $3,5(1,1)$ & $3,6(1,1)$ & $3,9(1,0)$ & $0,3(1,3)$ & $<0,001$ \\
\hline \multicolumn{6}{|l|}{ Dominios } \\
\hline $\begin{array}{l}\text { Percepciones sobre el médico que trabaja en el } \\
\text { PNA (preguntas 1, 2, 3, } 5 \text { y 7; puntaje del } 5 \text { al 25) }\end{array}$ & $12,7(3,6)$ & $13,0(3,4)$ & $15,1(3,8)$ & $2,1(4,0)$ & $<0,001$ \\
\hline $\begin{array}{l}\text { Percepciones sobre el trabajo asistencial en el PNA } \\
\text { (preguntas } 8,9,10 \text { y } 11 \text {; puntaje del } 4 \text { al } 20 \text { ) }\end{array}$ & $12,8(2,8)$ & $13,3(2,7)$ & $14,7(3,0)$ & $1,5(3,4)$ & $<0,001$ \\
\hline $\begin{array}{l}\text { Percepciones sobre las consecuencias económicas } \\
\text { de trabajar en el PNA (preguntas } 4 \text { y 6; puntaje del } \\
2 \text { al 10) }\end{array}$ & $6,8(1,8)$ & $7,1(1,8)$ & $7,0(2,0)$ & $-0,1(2,2)$ & 0,447 \\
\hline Total & $32,3(6,5)$ & $33,3(5,9)$ & $36,8(6,9)$ & $3,5(6,7)$ & $<0,001$ \\
\hline
\end{tabular}

PNA: Primer nivel de atención.

${ }^{a}$ Prueba t pareada.

ser atractivo para laborar en el PNA durante el SERUMS; se ha descrito que una de las motivaciones para el trabajo y retención del personal médico en zonas rurales y remotas son los incentivos económicos ${ }^{(8,19)}$.
La mayor percepción negativa hacia el trabajo en el PNA se encontró en los ítems «¿considera que la formación académica que ha recibido en la universidad está orientada más a un trabajo hospitalario que del PNA?», «¿considera que el 
Tabla 3. Factores asociados a la percepción de los médicos sobre el trabajo en el primer nivel de atención en la encuesta basal $(n=723)$.

\begin{tabular}{|c|c|c|}
\hline \multirow[b]{2}{*}{ Variables } & \multicolumn{2}{|c|}{ Encuesta basal } \\
\hline & $\begin{array}{l}\text { Modelo crudo } \\
\beta \text { (IC 95\%) }\end{array}$ & $\begin{array}{l}\text { Modelo ajustado } \\
\beta(\text { IC 95\%) }\end{array}$ \\
\hline \multicolumn{3}{|l|}{ Edad (años) } \\
\hline$\leq 25$ & Ref. & \\
\hline 26 & $-0,42(-1,78$ a 0,94$)$ & - \\
\hline$\geq 27$ & $-0,85(-1,92$ a 0,22$)$ & - \\
\hline \multicolumn{3}{|l|}{ Sexo } \\
\hline Masculino & Ref. & \\
\hline Femenino & $-0,18(-1,14$ a 0,77$)$ & - \\
\hline \multicolumn{3}{|c|}{ Ubicación de la universidad de procedencia } \\
\hline Ciudad de Lima & Ref. & Ref. \\
\hline Otras ciudades del Perú & $-1,79(-3,30 \mathrm{a}-0,27)$ & $-0,42(-2,41$ a 1,56$)$ \\
\hline Extranjero & $-0,42(-3,75$ a 2,91$)$ & $3,35(-0,98$ a 7,67$)$ \\
\hline \multicolumn{3}{|l|}{ Lugar de nacimiento } \\
\hline Ciudad de Lima & Ref. & Ref. \\
\hline Otras ciudades & $-0,69(-1,65$ a 0,28$)$ & $0,01(-1,19$ a 1,22$)$ \\
\hline \multicolumn{3}{|c|}{ Estudió otra carrera antes de Medicina } \\
\hline No & Ref. & Ref. \\
\hline Sí & $-1,85(-3,60$ a $-0,09)$ & $-0,55(-2,65$ a 1,55$)$ \\
\hline \multicolumn{3}{|c|}{ Nivel básico de quechua o aimara } \\
\hline No & Ref. & Ref. \\
\hline Sí & $-2,44(-3,95 \mathrm{a}-0,93)$ & $-2,67(-4,29 a-1,06)$ \\
\hline \multicolumn{3}{|l|}{ Familia dependiente } \\
\hline No & Ref. & Ref. \\
\hline Sí & $-1,55(-3,01$ a $-0,09)$ & $-1,75(-3,48$ a $-0,01)$ \\
\hline \multicolumn{3}{|l|}{ Familiares médicos } \\
\hline No & Ref. & \\
\hline Sí & $0,24(-0,71$ a 1,19$)$ & - \\
\hline \multicolumn{3}{|c|}{ Participación en actividades de PNA durante el pregrado } \\
\hline No & Ref. & Ref. \\
\hline Sí & $-1,55(-3,62$ a 0,53$)$ & $-1,86(-4,23$ a 0,51$)$ \\
\hline
\end{tabular}

PNA: Primer nivel de atención.

a Ajustado por edad, universidad de procedencia, lugar de nacimiento, otras carreras universitarias, nivel básico de quechua y aimara, familia dependiente y participación en actividades de PNA durante el pregrado.

trabajo en el PNA es muy limitado en relación con la actividad hospitalaria?», "¿considera que si trabajase en el PNA en su país, no podría satisfacer sus necesidades económicas?». Otros estudios mencionan que una de las razones por las que el médico no tiene interés en laborar en el PNA es porque su labor profesional en zonas alejadas es incompatible con el perfeccionamiento profesional. Por este motivo consideran que el trabajo en el PNA sería solo para médicos recientemente graduados, es decir, un ciclo de transición antes de que laboren en la zona urbana ${ }^{(8,9)}$. En el Perú, la participación en el SERUMS es un requisito indispensable para optar por una especialización ${ }^{(4)}$, por lo que el médico con interés o no, por el trabajo en el PNA, debe participar en este programa para continuar con su perfeccionamiento profesional.

El único ítem que disminuyó su puntaje promedio (es decir, en el cual la percepción mejoró al realizar el SERUMS) fue el considerar que el médico en PNA recibe un pago menor comparado al médico hospitalario. Esto puede deberse a que el sueldo en el SERUMS es superior al de un médico que labora en zonas urbanas. En general, se ha reportado que los médicos peruanos perciben que su salario es bajo ${ }^{(20)}$. Cuando la densidad de médicos en la región es baja, es esperable que reciban un mayor salario; asimismo, los médicos esperan recibir un mayor sueldo si trabajan en zonas rurales ${ }^{(10)}$, como compensación por la dificultad y las carencias de dichas zonas ${ }^{(8)}$. Si bien, el incentivo económico es importante para laborar en el PNA, los médicos también buscan otros beneficios, como capacitarse desde su zona de trabajo o en zonas aledañas, recibir becas o bonificaciones por trabajar en estas zonas ${ }^{(7-9)}$. Se ha descrito que el empleo de incentivos mixtos (económicos y no económicos) en lugar de únicamente económicos, incrementa el interés de los profesionales sanitarios por laborar en el PNA ${ }^{(21-23)}$. Este tipo de incentivos ha sido recomendado por la Organización Mundial de la Salud para mejorar la retención 
Tabla 4. Factores asociados a la diferencia de puntaje en la percepción de los médicos sobre el trabajo en el primer nivel de atención entre la encuesta basal y la encuesta de seguimiento $(n=215)$.

\begin{tabular}{|c|c|}
\hline Variables & $\begin{array}{l}\text { Modelo crudo } \\
\beta(\text { IC } 95 \%)\end{array}$ \\
\hline \multicolumn{2}{|l|}{ Edad en años } \\
\hline$\leq 25$ & Ref. \\
\hline 26 & $1,56(-0,90$ a 4,01$)$ \\
\hline$\geq 27$ & $0,32(-1,82$ a 2,45$)$ \\
\hline \multicolumn{2}{|l|}{ Sexo } \\
\hline Masculino & Ref. \\
\hline Femenino & $-1,11(-2,95$ a 0,73$)$ \\
\hline \multicolumn{2}{|l|}{ Universidad de procedencia } \\
\hline Ciudad de Lima & Ref. \\
\hline Otras ciudades del Perú & $-0,19(-4,94$ a 4,57$)$ \\
\hline Extranjero & $-1,94(-11,31$ a 7,44$)$ \\
\hline \multicolumn{2}{|l|}{ Lugar de nacimiento } \\
\hline Ciudad de Lima & Ref. \\
\hline Otras ciudades & $-1,21(-3,07$ a 0,65$)$ \\
\hline \multicolumn{2}{|c|}{ Estudió otra carrera antes de medicina } \\
\hline No & Ref. \\
\hline Sí & $1,29(-2,36$ a 4,94$)$ \\
\hline \multicolumn{2}{|c|}{ Nivel básico de quechua o aimara } \\
\hline No & Ref. \\
\hline Sí & $-0,14(-2,96$ a 2,69$)$ \\
\hline \multicolumn{2}{|l|}{ Familia dependiente } \\
\hline No & Ref. \\
\hline Sí & $1,60(-2,04$ a 5,24$)$ \\
\hline \multicolumn{2}{|l|}{ Familiares médicos } \\
\hline No & Ref. \\
\hline Sí & $1,19(-0,61$ a 2,98$)$ \\
\hline \multicolumn{2}{|c|}{$\begin{array}{l}\text { Participación en actividades de PNA durante } \\
\text { el pregrado }\end{array}$} \\
\hline No & Ref. \\
\hline Sí & $-2,05(-6,12$ a 2,01$)$ \\
\hline \multicolumn{2}{|c|}{ Tipo de establecimiento de salud } \\
\hline Ministerio de Salud & Ref. \\
\hline Otros & $-1,30(-3,97$ a 1,37$)$ \\
\hline \multicolumn{2}{|c|}{ Categoría del establecimiento de salud } \\
\hline I-1 & Ref. \\
\hline I-2 & $-1,27(-3,61$ a 1,07$)$ \\
\hline I-3 o I-4 & $0,72(-1,90$ a 3,34$)$ \\
\hline \multicolumn{2}{|c|}{ Jefatura del establecimiento de salud } \\
\hline No & Ref. \\
\hline Sí & $0,20(-1,65$ a 2,04$)$ \\
\hline
\end{tabular}

PNA: Primer nivel de atención.

de los profesionales en el PNA de zonas remotas y rurales ${ }^{(23)}$.

El deterioro en la perspectiva de los serumistas hacia el trabajo en el PNA al realizar el SERUMS puede deberse a una serie de problemas reportados en esta población durante su participación en este programa, como recibir agresiones verbales, psicológicas y físicas ${ }^{(24)}$, problemas administrativos y de clima laboral ${ }^{(25,26)}$, exposición a accidentes de tránsito durante su servicio y muerte durante el traslado de pacientes ${ }^{(5,27)}$, y no contar con una cobertura de aseguramiento adecuada ${ }^{(28)}$. Además, la carencia de insumos, tecnología y problemas en la atención de los pacientes limita la posibilidad del médico de brindar una atención adecuada ${ }^{(9)}$. Al respecto, una reciente modificación de la Ley de Servicio Rural y Urbano Marginal de Salud-SERUMS señala que, a partir del 2019, los serumistas, durante el año de servicio, contarán con un seguro contra accidentes personales que cubre muerte accidental, invalidez permanente, gastos de curación y gastos de sepelio ${ }^{(5)}$; esto podría mejorar la percepción de los médicos sobre el PNA durante el SERUMS.

En el Perú, se ha incrementado la oferta de plazas remuneradas para el SERUMS, tanto en el MINSA, en el Seguro Social y en las fuerzas armadas. Para el 2015, el 98\% de distritos del quintil 1 (quintil más pobre) tenían laborando médicos serumistas ${ }^{(6)}$. La política de asignar recursos humanos al PNA busca mejorar la distribución de profesionales de salud en las zonas remotas y rurales ${ }^{(5)}$. Sin embargo, se debe considerar que el médico serumista es un profesional recientemente egresado y que el SERUMS sería su primera experiencia profesional, lo que le generaría desafíos y dificultades, como gestionar los establecimientos del PNA, situación descrita previamente ${ }^{(29,30)}$. Este último punto resalta la necesidad de evaluar las competencias de los profesionales recién egresados respecto al PNA, dada la brecha existente en la formación en el aula (que está más orientada al ámbito hospitalario) para mejorar la formación de estos profesionales y su inserción al mundo laboral ${ }^{(6)}$.

Investigaciones realizadas en estudiantes de pregrado mencionan que la experiencia previa en el PNA está asociada a una mayor intención de trabajo en el este nivel de atención; se describe que trabajar y residir varios meses en zonas rurales y tener dificultades laborales disminuyen la intención del trabajo posterior en el PNA ${ }^{(10,12,15)}$. Estos hallazgos en población estudiantil difieren con lo encontrado en este estudio, debido a que la experiencia en el PNA evaluada en los estudiantes sería más un evento vivencial tipo «campamento estudiantil», de corta duración (pocos días), en donde experimentan algunas situaciones de la labor diaria en el PNA, lo que no se puede denominar un auténtico trabajo en el PNA. Lo descrito se puede ratificar por múltiples reportes que describen diversos tipos de dificultades durante la labor en el PNA ${ }^{(24,30)}$. Si bien se describen experiencias de la población estudiantil en el PNA, consideramos que se deben tener definiciones más rigurosas para poder considerar el desarrollo de una actividad, como una experiencia de trabajo en este nivel de atención (por ejemplo, rotar en un servicio de cirugía no puede catalogarse como experiencia quirúrgica, sino como una rotación en un área quirúrgica).

Entre las limitaciones de este estudio se deben mencionar las siguientes: 1) Al ser un análisis secundario de una base de datos, se limita la disponibilidad de otras variables como la seguridad, la disponibilidad de equipos, la capacitación continua, entre otras, que podrían influir en la percepción de 
los serumistas sobre el trabajo en el PNA. 2) Existió un alto porcentaje de participantes que no respondió la encuesta de seguimiento $(72,4 \%)$, lo cual era esperable considerando que se usó una encuesta en línea (dificultad de acceso y escasa cobertura de Internet en los establecimientos de salud donde se realizó el SERUMS). Asimismo, el bajo número de participantes que respondieron la encuesta de seguimiento podría haber influido en la no identificación de variables asociadas a la diferencia de puntaje en la percepción de los serumistas sobre trabajo en el PNA. Sin embargo, el empleo de esta escala ofrece ciertas ventajas, como la posibilidad de responder la encuesta en cualquier momento donde se tenga acceso a Internet, en los días libres o cuando realizaban trámites en las ciudades.

A pesar de estas limitaciones, los resultados muestran un panorama sobre la intención del trabajo en el PNA de médicos recientemente graduados, antes y durante el SERUMS, programa que, en muchos casos, es la primera y única experiencia de trabajo en el PNA de los médicos peruanos.

\section{REFERENCIAS BIBLIOGRÁFICAS}

1. World Health Organization. Health workforce: data and statistics [Internet]. Ginebra: WHO; 2018 [citado el 22 de marzo del 2019]. Disponible en: https://www.who.int/hrh/statistics/en/.

2. Ministerio de Salud. Información de Recursos Humanos en el Sector Salud [Internet]. Lima: MINSA; 2017 [citado el 14 de junio del 2020]. Disponible en: http://bvs.minsa.gob.pe/local/MINSA/4559.pdf.

3. Ministerio de Salud. Plan Nacional de Fortalecimiento del Primer Nivel de Atención 2011-2021 [Internet]. Lima: MINSA; 2011 [citado el 14 de junio del 2020]. Disponible en: http://bvs.minsa.gob.pe/local/minsa/1620.pdf.

4. Congreso de la República. Decreto Ley 23330, Ley del Servicio Rural y Urbano Marginal de Salud - SERUMS [Internet]. Lima: Congreso de la República; 1981 [citado el 14 de junio del 2020]. Disponible en: https:// cdn.www.gob.pe/uploads/document/file/520483/ley-del-servicio-ruraly-urbano-marginal-de-salud-ley-n-23330.pdf.

5. Congreso de la República. Decreto Supremo 007-2008SA, aprueban modificaciones del reglamento de la ley No 23330, Ley de Servicio Rural y Urbano Marginal de Salud-SERUMS [Internet]. Lima: Congreso de la Republica; 2008 [citado el 22 de marzo del 2020]. Disponible en: https://cdn.www.gob.pe/uploads/document/file/277149/248433_DS0072008SA.pdf20190110-18386-1i4lx2a.pdf.

6. Ministerio de Salud. Evaluación de competencias a los profesionales médicos, obstetras y enfermeros del Servicio Rural y Urbano Marginal en Salud SERUMS [Internet]. Lima: MINSA; 2008 [citado el 22 de marzo del 2020]. Disponible en: http://bvs.minsa.gob.pe/local/MINSA/4337.pdf.

7. Huicho L, Miranda JJ, Diez-Canseco F, Lema C, Lescano AG, Lagarde $\mathrm{M}$, et al. Job preferences of nurses and midwives for taking up a rural job in Peru: a discrete choice experiment. PloS one. 2012;7(12):e50315. doi: 10.1371/journal.pone.0050315.

8. Huicho L, Canseco FD, Lema C, Miranda JJ, Lescano AG. Incentivos para atraer y retener personal de salud de zonas rurales del Perú: un estudio cualitativo. Cad Saude Publica. 2012;28:729-39. doi: 10.1590/s0102311×2012000400012.

9. Huaynate CFA, Travezaño MJP, Correa M, Malpartida HM, Oberhelman $\mathrm{R}$, Murphy LL, et al. Diagnostics barriers and innovations in rural areas: insights from junior medical doctors on the frontlines of rural care in Peru. BMC Health Serv Res. 2015;15(1):454. doi: 10.1186/s12913-015-1114-7.
La percepción de los médicos que participan en el SERUMS sobre el trabajo en el PNA se deteriora después de un periodo de 8-12 meses luego de iniciar el servicio. Las estrategias que busquen fomentar el interés del médico en el PNA, incluyendo al médico serumista, podrían incluir nuevos incentivos mixtos (económicos y no económicos). De igual forma, una malla curricular orientada a la atención primaria en salud, incluyendo rotaciones en el PNA, podría incrementar el interés del profesional médico (o futuro profesional) en el trabajo en este nivel de atención.

Contribuciones de autoría: FIB, ATR, CMA concibieron y diseñaron el estudio. GBQ y LFMH recopilaron los datos. ATR procesó los datos. Todos los autores participaron en la interpretación de los datos, redacción del manuscrito y aprobación de la versión final.

Financiamiento: Autofinanciado.

Conflictos de interés: Los autores declaran no tener conflictos de interés.

Agradecimientos: Al Comité del Médico Joven del Colegio Médico del Perú, gestión 2015-2016, por facilitar la aplicación de este estudio.

10. Mayta-Tristán P, Mejia CR, Riega-Lopez P, Rojas-Mezarina L, Posso M, Mezones-Holguín E. Proyección de trabajo en el interior del país y factores asociados en médicos recién colegiados de Lima, Perú 2010. Rev Peru Med Exp Salud Publica. 2011;28:186-93. doi: 10.17843/ rpmesp.2011.282.483.

11. Mayta-Tristán P, Mezones-Holguín E, Pereyra-Elías R, Montenegro-Idrogo JJ, Mejia CR, Dulanto-Pizzorni A, et al. Diseño y validación de una escala para medir la percepción sobre el trabajo en el primer nivel de atención en estudiantes de medicina de Latinoamérica. Rev Peru Med Exp Salud Publica. 2013;30(2):190-6. doi: 10.17843/rpmesp.2013.302.190.

12. Tarqui-Mamani CB, Sanabria Rojas HA, Zárate Cárdenas E. Expectativas de laborar en el primer nivel de atención de salud de los estudiantes de una facultad de medicina de Lima, Perú. An Fac Med. 2015;76(1):57-2. doi: 10.15381/anales.v76i1.11076.

13. Taype-Rondán Á, Inga-Berrospi F, Casiano Celestino R, Bastidas F. Percepción de médicos recién egresados sobre las habilidades clínicas adquiridas durante el pregrado en Lima, Perú. Rev Med Chil. 2015;143(4):540-2. doi: 10.4067/S0034-98872015000400019.

14. Rivas-Nieto AC, Curioso WH, Guillén C. Participación estudiantil en proyectos de intervención rural en salud: la experiencia IRIS-X en Perú. Rev Peru Med Exp Salud Publica. 2009;26(3):387-94. doi: 10.17843/ rpmesp.2009.263.1392.

15. Escalante-Romero L, Cueva-Chávez L, Linares-Reyes E, Blossiers-Mazzini C. Experiencias de trabajo en actividades en atención primaria de salud realizadas por estudiantes de medicina de Perú. CIMEL. 2011;15(1):14-18.

16. Rabinowitz HK, Diamond JJ, Markham FW, Paynter NP. Critical factors for designing programs to increase the supply and retention of rural primary care physicians. JAMA. 2001;286(9):1041-8. doi: 10.1001/ jama.286.9.1041.

17. Brooks RG, Walsh M, Mardon RE, Lewis M, Clawson A. The roles of nature and nurture in the recruitment and retention of primary care physicians in rural areas: a review of the literature. Acad Med. 2002;77(8):790-8. doi: 10.1097/00001888-200208000-00008.

18. Rabinowitz HK, Diamond JJ, Markham FW, Hazelwood CE. A program to increase the number of family physicians in rural and underserved 
areas: impact after 22 years. JAMA. 1999;281(3):255-60. doi: 10.1001/ jama.281.3.255.

19. Buykx P, Humphreys J, Wakerman J, Pashen D. Systematic Review of Effective Retention Incentives for Health Workers in Rural and Remote Areas: Towards Evidence-Based Policy. Aust J Rural Health. 2010 Jun;18(3):102-9. doi: 10.1111/j.1440-1584.2010.01139.x.

20. Jumpa M, Jan S, Mills A. The role of regulation in influencing income-generating activities among public sector doctors in Peru. Hum Resour Health. 2007;5:5. doi: 10.1186/1478-4491-5-5.

21. Blaauw D, Erasmus E, Pagaiya N, Tangcharoensathein V, Mullei K, Mudhune S, et al. Policy interventions that attract nurses to rural areas: a multicountry discrete choice experiment. Bull World Health Organ. 2010;88:350-6. doi: 10.2471/BLT.09.072918.

22. Kruk ME, Johnson JC, Gyakobo M, Agyei-Baffour P, Asabir K, Kotha SR, et al. Rural practice preferences among medical students in Ghana: a discrete choice experiment. Bull World Health Organ. 2010;88:333-41. doi: 10.2471/BLT.09.072892.

23. World Health Organization. Increasing access to health workers in remote and rural areas through improved retention: global policy recommendations [Internet]. Ginebra: WHO; 2010 [citado el 22 de marzo del 2019]. Disponible en: https://www.who.int/hrh/retention/guidelines/en.

24. Mejia CR, Inga-Berrospi F, Lezama CA, García AAQ. Problemas laborales en plazas del Servicio Rural Urbano-Marginal en Salud (SERUMS): au- to-reporte de los médicos. Acta Méd Peru. 2016;33(1):82-3. doi: 10.35663/ amp.2016.331.23.

25. Mejía CR, Quiñones-Laveriano DM. SERUMS y la migración de médicos: a propósito de una cohorte de médicos de Lima. Rev Peru Med Exp Salud Publica. 2015;32(2):405-6. doi: 10.17843/rpmesp.2015.322.1647.

26. Taype-Rondan Á, Vidal-Torres MI, Chung-Delgado K, Maticorena-Quevedo J, Mayta-Tristán P. Problems perceived and experienced by health professionals rendering social service in Ancash, Peru. 2015. Rev Fac Med. 2017;65(3):441-6. doi: 10.15446/revfacmed.v65n3.59055.

27. Galán-Rodas E, Díaz-Vélez C, Villena J, Maguiña C. Mortalidad de médicos que realizan el servicio rural (SERUMS) en Perú, 2006-2009. Rev Peru Med Exp Salud Publica. 2010;27:483-4. doi: 10.17843/rpmesp.2010.273.1513.

28. Mejia CR, Quiñones-Laveriano DM, Espinoza KG, Quezada-Osoria C. Deficiente cobertura de aseguramiento a médicos durante el servicio rural y urbano-marginal en Perú. Rev Peru Med Exp Salud Publica. 2013;30:220-3. doi: 10.17843/rpmesp.2013.302.194.

29. Motta F, Frisancho A. La experiencia serums y la formación profesional. Rev Peru Epidemiol. 1992;5(2):24-8.

30. Valencia T, León B, Lezarneta U, Vidal M. Principales dificultades del profesional de salud encontrados durante el Serums en el departamento de Ancash, zona sierra. 2008. Rev. Aporte Santiaguino, 2011;4(1):118-22. doi: 10.32911/as.2011.v4.n1.537. 